\title{
How adjustment could affect internal and marginal adaptation of CAD/CAM crowns made with different materials
}

\author{
Mahya Hasanzade', Mohammad Moharrami², Marzieh Alikhasi²* \\ ${ }^{1}$ Dental Research Center, Dentistry Research Institute, Department of Prosthodontics, School of Dentistry, Tehran University of \\ Medical Sciences, Tehran, Iran \\ ${ }^{2}$ Dental Research Center, Dentistry Research Institute, Tehran University of Medical Sciences, Tehran, Iran
}

\begin{abstract}
PURPOSE. Recently introduced hybrid and reinforced glass ceramic computer-aided design/computer-aided manufacturing (CAD/CAM) materials have been used for full-coverage restorations. However; the effect of adjustment and type of materials on internal and marginal adaptation are unknown. This study aimed to evaluate and compare the marginal and internal adaptations of crowns made of three different CAD/CAM materials before and after adjustment. MATERIALS AND METHODS. One acrylic resin maxillary first molar was prepared and served as the master die. Thirty-six restorations were fabricated using CAD/CAM system (CEREC Omnicam, $\mathrm{MCXL}$ ) with three materials including lithium disilicate (IPS e.max CAD), zirconia-reinforced lithium silicate (Suprinity), and hybrid ceramic (Enamic). Internal and marginal adaptations were evaluated with the reference point matching technique before and after adjustment. The data were analyzed using mixed ANOVA considering $\alpha=.05$ as the significance level. RESULTS. The effect of adjustment and its interaction with the restoration material were significant for marginal, absolute marginal, and occlusal discrepancies $(P<.05)$. Before adjustment, Suprinity had lower marginal discrepancies than IPS e.max CAD $(P=.18)$ and Enamic $(P=.021)$; though no significant differences existed after adjustment. CONCLUSION. Within the limitations of this study, crowns fabricated from IPS e.max CAD and Suprinity resulted in slightly better adaptation compared with Enamic crowns before adjustment. However, marginal, axial, and occlusal discrepancies were similar among all materials after the adjustment. [J Adv Prosthodont 2020;12:344-50]
\end{abstract}

KEYWORDS: Ceramic restoration; Computer-aided design/computer-aided manufacturing (CAD/CAM); Internal fit; Marginal fit; Adjustment

\section{INTRODUCTION}

Full-coverage crowns are one of the most common fixed restorations for reconstruction of severely damaged teeth. ${ }^{1}$ Metal-ceramic restorations were the gold standard for restoring teeth for many years. However, all-ceramic restora-

\footnotetext{
Corresponding author:

Marzieh Alikhasi

Department of Prosthodontics, School of Dentistry, Tehran University of Medical Sciences, North Kargar street, Tehran, Iran

Tel. +989122014160: e-mail, malikhasi@razi.tums.ac.ir

Received February 22, 2020 / Last Revision October 24, 2020 / Accepted November 5, 2020

(C) 2020 The Korean Academy of Prosthodontics

This is an Open Access article distributed under the terms of the Creative Commons Attribution Non-Commercial License (http://creativecommons. org/licenses/by-nc/4.0) which permits unrestricted non-commercial use, distribution, and reproduction in any medium, provided the original work is properly cited.
}

tions are gradually substituting the metal-based restorations due to the growing demand of a high-quality esthetic. Due to the elimination of the metal element, there would be a better distribution of the reflected light, which in turn provide the improved shade matching in all-ceramic restorations. $^{2}$

Similar to any newly developing technique, computeraided design/computer-aided manufacturing (CAD/CAM) crowns restorations still need evaluations to be applied successfully in the clinical settings. One of the important factors determining the survival of the restorations is crown adaptation. Improperly fitted restorations lead to periodontal disease, secondary caries, and ultimately failure of the restorations. ${ }^{3,4}$ Choosing the right fabrication material can play a significant role in various characteristics of final restorations. CAD/CAM crowns can be manufactured from a variety of materials including composite resins, ceramics, 
reinforced ceramics, sintered-alumina, and hybrid ceramics. ${ }^{5,6}$ One of the materials which have been broadly used in the last decade is lithium disilicate glass-ceramic (LDGC) due to its esthetic privilege. ${ }^{7-9}$ However, the survival rate of restorations fabricated from LDGC is still questionable. ${ }^{10}$ Therefore, modified ceramics such as zirconia-reinforced lithium silicate (ZLS) and polymer-infiltrated ceramic network (PICN) have been developed to address some of these deficiencies.

Vita Suprinity, a type of ZLS material, is enriched with zirconia $\left(\mathrm{ZrO}_{2}, 10 \mathrm{wt} \%\right)^{11}$ and provides the strength of zirconia with the translucency of glass ceramics. ZLS restorations can be polished more efficiently than LDGC restorations due to smaller crystal size $(6 \mu \mathrm{m}$ vs. $1 \mu \mathrm{m}) \cdot{ }^{12}$ Moreover, except for the lower brittleness index of LDGC which indicates better machinability, ZLS have shown superiority to LDGC regarding most mechanical properties such as fracture toughness, flexural strength, elastic modulus, and hardness. ${ }^{13}$

One of the commercialized PICN materials is Vita Enamic, which is obtained by infiltrating an acrylate polymer $(14 \mathrm{wt} \%)$ into a feldspathic ceramic matrix (86 wt \%), resulting in a fully integrated network. PICNs have better machinability compared with lithium disilicates due to lower flexural modulus and Vickers hardness. ${ }^{14,15}$ The main advantage of PICNs compared with other ceramics is a close elastic modulus (30 GPa,) to dentine (20 Gpa). ${ }^{16,17}$

High marginal accuracy and an adequate internal fit are the major predictors of successful clinical performance. ${ }^{18,19}$ When choosing an all-ceramic restoration, obtaining a marginal adaptation in a clinically acceptable range, which is less than $120 \mu \mathrm{m}$, is of major importance. Besides the type of material, adjustment of restorations before cementation is a matter of great importance and can determine the final internal and marginal discrepancies. ${ }^{20-22}$

This study aimed to evaluate and compare the marginal and internal adaptations of crowns made of three different CAD/CAM materials before and after adjustment. The first null hypothesis was that there would be no difference between LDGC (IPS e.max CAD), ZLS (Vita Suprinity), and PICN (Vita Enamic) regarding marginal and internal adaptation of full-coverage restorations. The second null hypothesis was that adjustment would not affect marginal and internal adaptation of full-coverage crowns.

\section{MATERIALS AND METHODS}

A maxillary first molar typodont (Nissin Dental Prod. Inc., Tokyo, Japan) was considered as the master model and was mounted in an aluminum base with an accurate geometric index, which was used to determine $\mathrm{X}, \mathrm{Y}$, and $\mathrm{Z}$ axis. The tooth model was secured by fast-cure acrylic resin (Fastray, Harry J. Bosworth Co., Skokie, IL, USA) so that the tooth was embedded up to $2 \mathrm{~mm}$ below the hypothetical cementoenamel junction and scanned by an intraoral scanner (CEREC; Omnicam Sirona Dental System, Bensheim, Germany) to record the outer surface. Then the standard preparation for an all-ceramic with $2 \mathrm{~mm}$ reduction of the occlusal surface and $1 \mathrm{~mm}$ reduction of all the axial walls with a deep chamfer finish line was performed using a round end taper diamond bur (806314290 coarse, Jota, Ruthi, Switzerland). A similar round end taper fine diamond bur (806314290 fine Jota, Ruthi, Switzerland) was used for smoothing the prepared surface. The completed tooth preparation was checked by two clinicians who had not performed the preparation to verify the standard preparation of crown for full ceramic restoration without undercut (Fig. 1).

In order to create the die scan, the prepared typodont was scanned using an intraoral scanner (CEREC; Omnicam Sirona Dental System, Bensheim, Germany). Using the biogeneric copy mode of the CEREC device, the original scan of the occlusal surface of the master model was transferred to the milled restorations. Based on the clinician's experience, $60 \mu \mathrm{m}$ and $10 \mu \mathrm{m}$ relief was considered for the crowns as the axial and marginal cement space accordingly. Overall, 36 crowns were obtained, and three groups of full contour crowns, each containing 12 samples, were considered based on the materials used: ZLS (Suprinity, VITA Zahnfabrik H. Rauter GmbH \& Co.KG, Bad Säckingen, Germany), PICN (Enamic, Zahnfabrik H. Rauter GmbH \& Co.KG, Bad Säckingen, Germany), and LDGC (IPS e.max CAD, Ivoclar Vivadent AG, Schaan, Liechtenstein). Restorations were milled using a milling unit CEREC MCXL (Dentsply Sirona, Bensheim, Germany) with $12 \mathrm{~s}$ step and cylinderpointed burs that were $1.3 \mathrm{~mm}$ and $1.8 \mathrm{~mm}$ in diameter, respectively. The milling burs were replaced when the changing instrument notification appeared on the system. ${ }^{22}$ For complete crystallization of IPS e.max CAD and Suprinity restorations, the milled crowns were fired in a furnace (CEREC Speed Fire, Dentsply Sirona, Bensheim, Germany).

After completing the fabrication process, the internal and marginal adaptations were measured using a reference point

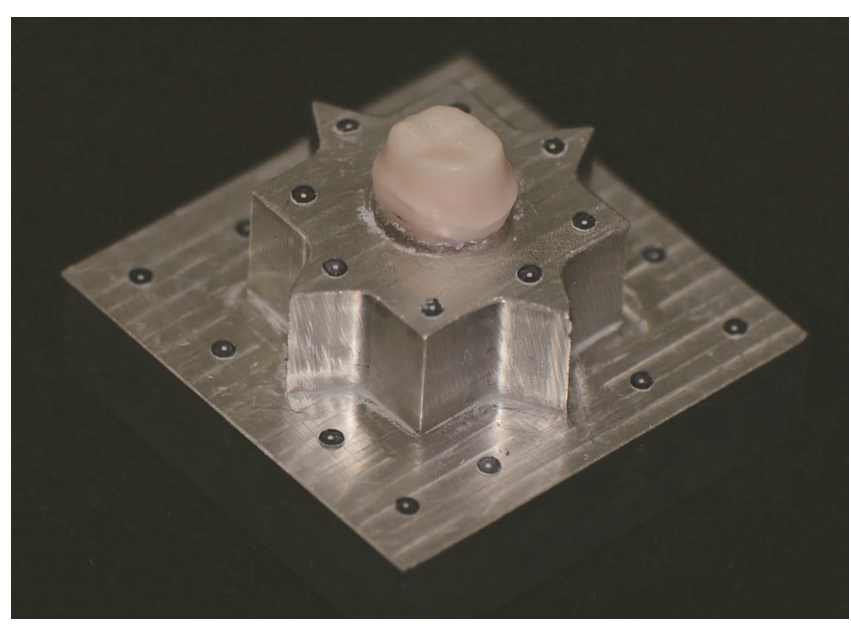

Fig. 1. Prepared tooth for full-coverage restoration. 
matching scan protocol, and the GOM software (GOM inspect v7.5, GOM mbH, Braunschweig, Germany) was used to analyze the dataset of each specimen. The scanning procedure was undertaken using a non-contact triple scanner (ATOS Core 5Mp 80 mm; Rev.02; GOM, Braunschweig; Germany), and four scans were obtained: 1 . The master die (prepared tooth) attached to the aluminum base, 2. The restorations sat on the master die in a correct position under 50 $\mathrm{N}$ load fixed by light body silicon (Speedex, Colten), 3. Hexshaped cylinder index was fixed on the occlusal surface of restoration, and all parts including base, tooth, restoration and hex-shaped index assembly were scanned, 4 . The restoration was removed from the tooth by the index being in its place, and then the inner and outer surfaces of the crown and hex-shaped index were scanned. After the initial measurement, a specialist adjusted all restorations using fine diamond bur (806 314199 514016, JOTA AG, Hirschensprungstrasse, Rüthi, Switzerland) with high speed handpiece and a blue fit checker material (Kettenbach, Eschenburg, Germany) under magnification of dental loop (HDL 2.5 Macro, Orascoptic, WI, USA). Adjustment continued until achievement of best seating possible according to the opinion of clinician. Measurements of the internal and marginal gap of adjusted restorations were repeated similarly to the initial procedure.

Mesh data were transferred into a defined coordinate system, and two sections were created in mesiodistal and buccolingual direction using intersecting edges of the star in the base to serve as a reference in order to have sections in the same position in all samples (Fig. 2). Measurements were done in the following three sites for buccal, lingual, mesial, and distal parts: marginal, axial, and occlusal. The perpendicular measurement from the internal surface of the restoration to the prepared tooth at margin was recorded as

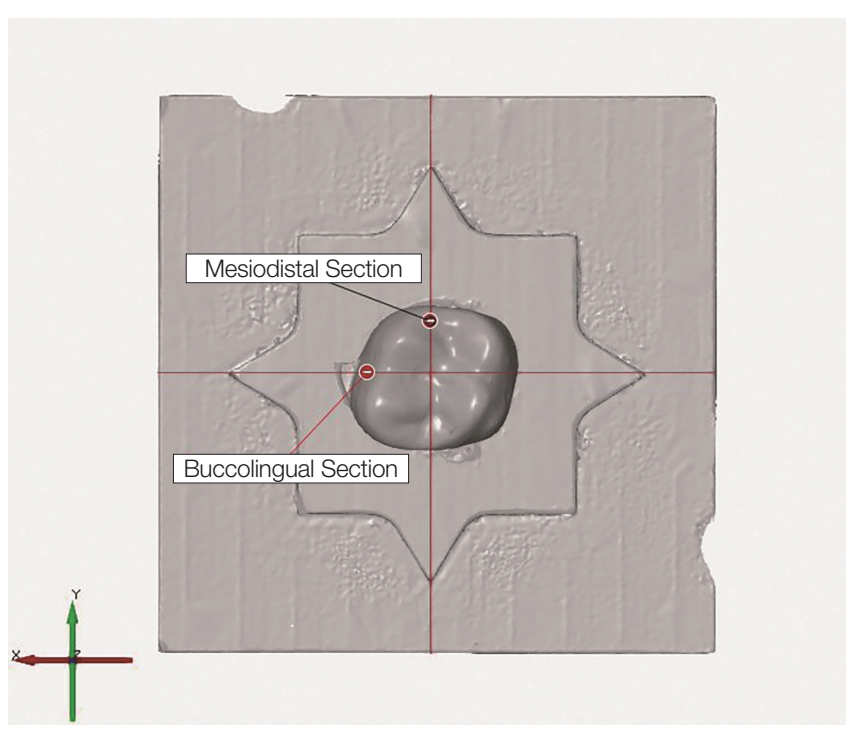

Fig. 2. The virtual model sectioned in mesiodistal and buccolingual directions. the marginal discrepancy, and the absolute marginal discrepancy was considered the combination of horizontal and vertical marginal gap. For axial discrepancy, the perpendicular distance from the internal surface of restoration to the prepared tooth at the mid-axial point was recorded (Fig. 3).

All of the analyses were performed using the Statistical Package for the Social Sciences (SPSS, IBM Corp. Version 22.0, Armonk, NY, USA). The quantitative data were presented by the mean and standard deviation. The KolmogorovSmirnov test was used to verify the normality of the collected data. Descriptive statistics including mean and standard deviation were reported for each group of material before and after adjustment. Mixed ANOVA was used to compare internal and marginal discrepancies among the three fabrication materials (between-subject factor) before and after adjustment (within-subject factor). The t-test was used to assess the simple effects of adjustment, and one-way ANOVA was used to determine the effect of fabrication material when there was a meaningful interaction between the main effects. A $P$ value less than .05 was considered to be statistically significant.

\section{RESULTS}

The descriptive results regarding internal and marginal discrepancies before and after adjustment for Suprinity, Enamic, and IPS e.max CAD have been presented in Table 1. The results showed that there was an interaction between the fabrication materials and adjustment for marginal $(P=$ .014), absolute marginal $(P=.038)$, and occlusal $(P=.021)$ discrepancies. Further analyses showed that the simple effect of adjustment was significant $(P<.001)$. Before the adjustment, the type of fabrication material had a significant

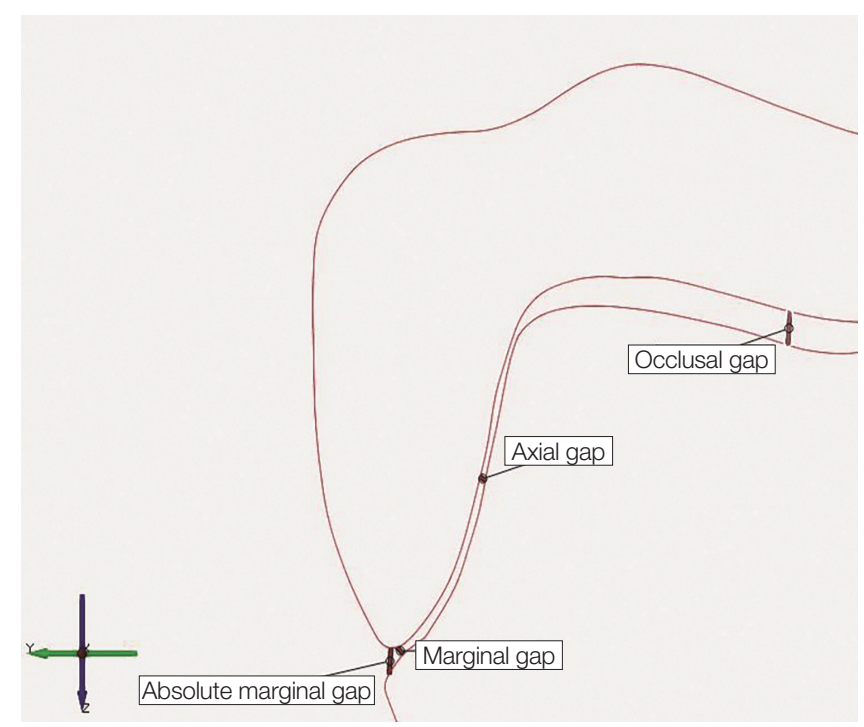

Fig. 3. A section of specimen. The lines represent the measurement discrepancies between the crown and the abutment tooth. 
Table 1. Mean and standard deviation of discrepancies $(\mu \mathrm{m})$ for the three materials at four sites before and after adjustment

\begin{tabular}{llcr}
\hline & Material & Mean and SD $(\mu \mathrm{m})$ before adjustment & Mean and SD $(\mu \mathrm{m})$ after adjustment \\
\hline Marginal discrepancy & e.max CAD & $187.125 \pm 50.859$ & $56.791 \pm 29.489$ \\
& Enamic & $190.090 \pm 67.684$ & $50.568 \pm 14.746$ \\
Absolute marginal discrepancy & Suprinity & $129.454 \pm 37.795$ & $62.590 \pm 25.689$ \\
& e.max CAD & $277.7083 \pm 67.692$ & $162.541 \pm 51.089$ \\
& Enamic & $356.295 \pm 118.661$ & $147.159 \pm 30.631$ \\
Occlusal discrepancy & Suprinity & $372.522 \pm 94.126$ & $177.045 \pm 60.685$ \\
& e.max CAD & $385.916 \pm 60.571$ & $248.500 \pm 64.740$ \\
Axial discrepancy & Enamic & $462.227 \pm 116.000$ & $219.818 \pm 35.744$ \\
& Suprinity & $475.409 \pm 94.446$ & $232.181 \pm 80.017$ \\
& e.max CAD & $57.885 \pm 17.750$ & $66.875 \pm 20.158$ \\
& Enamic & $76.920 \pm 17.500$ & $78.102 \pm 13.436$ \\
\hline
\end{tabular}

Table 2. Pairwise comparisons of discrepancy measurements among restorative materials

\begin{tabular}{|c|c|c|c|c|}
\hline Dependent variable & Restorative material & Mean difference & Std. error & Sig. \\
\hline Marginal discrepancy & e.max CAD vs Enamic & 1.41667 & 21.99622 & .998 \\
\hline \multirow[t]{2}{*}{ before adjust } & e.max CAD vs Suprinity & 63.64583 & 21.99622 & $.018^{*}$ \\
\hline & Suprinity vs Enamic & -62.22917 & 21.99622 & $.021^{*}$ \\
\hline Absolute marginal & e.max CAD vs Enamic & -92.47917 & 39.30448 & .062 \\
\hline \multirow[t]{2}{*}{ discrepancy before adjust } & e.max CAD vs Suprinity & -96.93750 & 39.30448 & $.049^{*}$ \\
\hline & Suprinity vs Enamic & 4.45833 & 39.30448 & .993 \\
\hline Occlusal discrepancy & e.max CAD vs Enamic & -96.04167 & 40.01870 & .056 \\
\hline \multirow[t]{2}{*}{ before adjust } & e.max CAD vs Suprinity & -92.95833 & 40.01870 & .066 \\
\hline & Suprinity vs Enamic & -3.08333 & 40.01870 & .997 \\
\hline Axial discrepancy & e.max CAD vs Enamic & -17.62500 & 21.99622 & $.039^{*}$ \\
\hline \multirow[t]{2}{*}{ before adjust } & e.max CAD vs Suprinity & -6.64583 & 21.99622 & .603 \\
\hline & Suprinity vs Enamic & -10.97917 & 6.87660 & .261 \\
\hline
\end{tabular}

impact on marginal $(P=.009)$, absolute marginal $(P=.031)$, and occlusal $(P=.035)$ discrepancies. Table 2 shows the pairwise comparisons in each site. On the contrary, after the adjustment, the type of fabrication material did not change the discrepancies significantly $(P>.05)$.

Regarding the axial discrepancy, there was no interaction between the fabrication material and adjustment $(P=.646)$. Unlike the three other sites, the adjustment had no significant effect on the discrepancies between the axial wall and restorations $(P=.125)$. On the contrary, the type of fabrication material had significant impact on the axial discrepancies $(P=.032)$. The pairwise comparison has been presented in Table 2.

\section{DISCUSSION}

Based on the results, the null hypotheses were partially rejected. Depending on the evaluated site, both adjustment and type of fabrication material could impact the discrepancies. With regards to the marginal, absolute marginal, and occlusal discrepancies, adjustment significantly decreased the discrepancies. Before the adjustment, there were differences between the fabrication materials, but the adjustment moderated the impact of fabrication materials since there were no significant differences after adjustment between Suprinity, Enamic, and IPS e.max CAD. With regards to the axial wall discrepancies, the effect of fabrication materials and adjustment were not 
dependent on each other, and while adjustment had no effect, the type of fabrication material significantly changed the discrepancies. Moreover, unlike the other three sites, axial discrepancies increased after adjustment. The unique trend observed for the axial discrepancy can probably be attributed to the removal of a layer from the axial walls during the adjustment.

Both clinical and laboratory related factors play roles in the internal and marginal adaptation of full-coverage restorations. ${ }^{23,24}$ That being said, based on our results, it seems chemical and microstructural properties specific to each material, which are translated into the mechanical properties, can impact the accuracy and precision of the CAD/ CAM restorations. However, these differences could be compensated after adjusting the restorations. Importantly, after the adjustment, the values for the marginal discrepancies fell below $120 \mu \mathrm{m}$, which is the clinically acceptable threshold. ${ }^{25}$

Based on the brittleness index values of Suprinity (2.84 $\left.\mu \mathrm{m}^{-1 / 2}\right)$, Enamic $\left(1.67 \mu \mathrm{m}^{-1 / 2}\right)$, and IPS e.max CAD $(2.72$ $\left.\mu \mathrm{m}^{-1 / 2}\right), 13,26$ it was anticipated that Enamic would result in the most accurate restorations. Moreover, when evaluated directly with the scanning electronic microscope (SEM) and digital microscope, Enamic was more machinable and less rough on the margins than IPS e.max CAD. ${ }^{27,28}$ However, the results indicated that Suprinity and IPS e.max CAD were at least as accurate as Enamic meaning that the milling machine could efficiently fabricate the restorations from all the three materials despite their hardness and machinability. These results may be partly due to the fact that all the values for brittleness index fell below $4.3 \mu \mathrm{m}^{-1 / 2}$, which is considered the acceptable cut-off point for machinability; therefore, all restorations could be milled accurately. ${ }^{29,30}$ Also, the marginal discrepancy for IPS e.max CAD was higher than Suprinity, which can be due to the high flexural strength of Suprinity which made it more resistant to the chipping and crack propagation. ${ }^{14}$

Overall marginal and axial discrepancies of all groups were less than absolute marginal and occlusal discrepancies. The higher range of absolute marginal discrepancies compared to marginal discrepancies might be due to the fact that absolute marginal discrepancy shows the total adaptation errors including the over-extension or under-extension of the restoration. ${ }^{31}$ The reason behind the high discrepancies at the occlusal site might be due to the overmilling, since milling instruments are available in limited diameters and occlusal surfaces that deducted following anatomical contours may not be milled accurately. High occlusal discrepancy can result in a thicker layer of the luting agent, which lead to incomplete seating of the restoration which in turn cause the tensile stresses in resin cement. ${ }^{32}$ Other studies that evaluated the marginal and internal adaptation of lithium disilicate reported similar high occlusal discrepancies. ${ }^{14,33}$

Until now, few studies have reported on the internal and marginal discrepancies of crowns considering material types. Yildirim et al. used similar fabrication materials and CAD/CAM device as used in this study, but reported differ- ent results. ${ }^{34}$ In our study, the overall internal and marginal discrepancies were higher probably due to higher cement space $(60 \mu \mathrm{m}$ vs. $40 \mu \mathrm{m})$. In the study by Yildirim et al., Enamic resulted in the best and IPS e.max CAD resulted in the worst internal and marginal discrepancies before adjustment. Measuring methods, which seems to be one of the most influential parameters in the reported results, were different between the two studies. ${ }^{34}$ In this study, a reference matching scan method was used to evaluate the restoration fit, which had 3D nature. On the contrary, Yildirim et al. used microcomputed tomography (m-CT) that employs $2 \mathrm{D}$ measurement.

Results of this study showed decreased marginal and internal gap after adjustment. The clinical adjustment may have undesirable consequence on the strength of ceramic. The results of previous studies on the effect of grinding have varied from detrimental effect to the ineffectiveness. ${ }^{35-37}$ A study suggested that effect of grinding may depend on the bur grit size, handpiece speed, wet or dry conditions, and mechanical properties of ceramic submitted to grinding. ${ }^{35}$ Curran et al. reported that grinding was quite detrimental to the IPS e.max CAD, with chip crack formation and a strength loss estimated at $42 \% .{ }^{35}$ However, another study showed that grinding did not affect the fracture resistance of Suprinity, Enamic, and IPS e.max CAD. ${ }^{36}$

Some notes should be considered when interpreting the results of this study. First, although the cement space was considered $60 \mu \mathrm{m}$, transferring this value with the same precision to the milled restorations might have been questionable. Second, parameters related to milling units such as the drill diameter, milling material, and application time, as well as the preparation design of the master model, can affect the precision. ${ }^{38}$ Rotary instruments with smaller diameters can yield improved details and more accurate restorations. Third, only one cement thickness was evaluated, but the internal fit may be influenced by altering the spacer thickness. Fourth, measuring the internal gap was done before cementation, which disregards the influence of the luting procedure and cement type on the internal gap. Fifth, the in vitro nature of this study prevents extending results to clinical settings, and whether these marginal and internal discrepancies are clinically acceptable remains unknown. The future studies also need to evaluate the relationship between the large occlusal gap and the fracture of the CAD/CAM restoration in the long-term period.

\section{CONCLUSION}

Within the limitations of this study, it could be concluded that crowns made of IPS e.max CAD and Suprinity resulted in slightly better adaptation compared to crowns made of Enamic. After the adjustment, the restorations fitted more accurately, and the differences among the materials were not significant. 


\section{ORCID}

Mahya Hasanzade https://orcid.org/0000-0002-9114-2471

Marzieh Alikhasi bttps://orcid.org/0000-0002-2527-728X

Mohammad Moharrami bttps://orcid.org/0000-0002-4364-7969

\section{REFERENCES}

1. Dolan TA, Gilbert GH, Duncan RP, Foerster U. Risk indicators of edentulism, partial tooth loss and prosthetic status among black and white middle-aged and older adults. Community Dent Oral Epidemiol 2001;29:329-40.

2. Volpato CÂ, Frede MC, Philippi AG, Petter CO. Ceramic materials and color in dentistry. Ceramic Materials, Ed. W. Wunderlich, InTech 2010:155-74.

3. Felton DA, Kanoy BE, Bayne SC, Wirthman GP. Effect of in vivo crown margin discrepancies on periodontal health. J Prosthet Dent 1991;65:357-64.

4. Jacobs MS, Windeler AS. An investigation of dental luting cement solubility as a function of the marginal gap. J Prosthet Dent 1991;65:436-42.

5. Conrad HJ, Seong WJ, Pesun IJ. Current ceramic materials and systems with clinical recommendations: a systematic review. J Prosthet Dent 2007;98:389-404.

6. Gracis S, Thompson VP, Ferencz JL, Silva NR, Bonfante EA. A new classification system for all-ceramic and ceramic-like restorative materials. Int J Prosthodont 2015;28:227-35.

7. Tysowsky G. The science behind lithium disilicate: Today's surprisingly versatile, esthetic \& durable metal-free alternative. Oral Health 2009;99:93-7.

8. Gehrt M, Wolfart S, Rafai N, Reich S, Edelhoff D. Clinical results of lithium-disilicate crowns after up to 9 years of service. Clin Oral Investig 2013;17:275-84.

9. Fasbinder DJ, Dennison JB, Heys D, Neiva G. A clinical evaluation of chairside lithium disilicate CAD/CAM crowns: a two-year report. J Am Dent Assoc 2010;141:10S-4S.

10. Pieger S, Salman A, Bidra AS. Clinical outcomes of lithium disilicate single crowns and partial fixed dental prostheses: a systematic review. J Prosthet Dent 2014;112:22-30.

11. Denry I, Kelly JR. Emerging ceramic-based materials for dentistry. J Dent Res 2014;93:1235-42.

12. Belli R, Wendler M, de Ligny D, Cicconi MR, Petschelt A, Peterlik H, Lohbauer U. Chairside CAD/CAM materials. Part 1: Measurement of elastic constants and microstructural characterization. Dent Mater 2017;33:84-98.

13. Elsaka SE, Elnaghy AM. Mechanical properties of zirconia reinforced lithium silicate glass-ceramic. Dent Mater 2016;32: 908-14.

14. Goujat A, Abouelleil H, Colon P, Jeannin C, Pradelle N, Seux D, Grosgogeat B. Mechanical properties and internal fit of 4 CAD-CAM block materials. J Prosthet Dent 2018;119:384-9.

15. He LH, Swain M. A novel polymer infiltrated ceramic dental material. Dent Mater 2011;27:527-34.

16. Ruse ND, Sadoun MJ. Resin-composite blocks for dental CAD/CAM applications. J Dent Res 2014;93:1232-4.

17. Ramos Nde C, Campos TM, Paz IS, Machado JP, Bottino MA, Cesar PF, Melo RM. Microstructure characterization and
SCG of newly engineered dental ceramics. Dent Mater 2016; 32:870-8.

18. Bottino MA, Valandro LF, Buso L, Ozcan M. The influence of cervical finish line, internal relief, and cement type on the cervical adaptation of metal crowns. Quintessence Int 2007; 38:e425-32.

19. Limkangwalmongkol P, Kee E, Chiche GJ, Blatz MB. Comparison of marginal fit between all-porcelain margin versus alumina-supported margin on Procera Alumina crowns. J Prosthodont 2009;18:162-6.

20. Johnson R, Verrett R, Haney S, Mansueto M, Challa S. Marginal gap of milled versus cast gold restorations. J Prosthodont 2017;26:56-63.

21. Tabata LF, de Lima Silva TA, de Paula Silveira AC, Ribeiro APD. Marginal and internal fit of CAD-CAM composite resin and ceramic crowns before and after internal adjustment. J Prosthet Dent 2020;123:500-5.

22. Azarbal A, Azarbal M, Engelmeier RL, Kunkel TC. Marginal fit comparison of CAD/CAM crowns milled from two different materials. J Prosthodont 2018;27:421-8.

23. Memari Y, Mohajerfar M, Armin A, Kamalian F, Rezayani V, Beyabanaki E. Marginal adaptation of CAD/CAM all-ceramic crowns made by different impression methods: A literature review. J Prosthodont 2019;28:e536-44.

24. Miyazaki T, Hotta Y, Kunii J, Kuriyama S, Tamaki Y. A review of dental CAD/CAM: current status and future perspectives from 20 years of experience. Dent Mater J 2009;28:44-56.

25. McLean JW, von Fraunhofer JA. The estimation of cement film thickness by an in vivo technique. Br Dent J 1971;131: 107-11.

26. Eldafrawy M, Nguyen JF, Mainjot AK, Sadoun MJ. A functionally graded PICN material for biomimetic CAD-CAM blocks. J Dent Res 2018;97:1324-30.

27. Chavali R, Nejat AH, Lawson NC. Machinability of CADCAM materials. J Prosthet Dent 2017;118:194-9.

28. Awada A, Nathanson D. Mechanical properties of resin-ceramic CAD/CAM restorative materials. J Prosthet Dent 2015; 114:587-93.

29. Chaysuwan D, Sirinukunwattana K, Kanchanatawewat K, Heness G, Yamashita K. Machinable glass-ceramics forming as a restorative dental material. Dent Mater J 2011;30:358-67.

30. Boccaccini A. Machinability and brittleness of glass-ceramics. J Mater Proc Tech 1997;65:302-4.

31. Holmes JR, Bayne SC, Holland GA, Sulik WD. Considerations in measurement of marginal fit. J Prosthet Dent 1989;62:4058.

32. May LG, Kelly JR, Bottino MA, Hill T. Effects of cement thickness and bonding on the failure loads of CAD/CAM ceramic crowns: multi-physics FEA modeling and monotonic testing. Dent Mater 2012;28:e99-109.

33. Zeltner M, Sailer I, Mühlemann S, Özcan M, Hämmerle CH, Benic GI. Randomized controlled within-subject evaluation of digital and conventional workflows for the fabrication of lithium disilicate single crowns. Part III: marginal and internal fit. J Prosthet Dent 2017;117:354-62.

34. Yildirim G, Uzun IH, Keles A. Evaluation of marginal and internal adaptation of hybrid and nanoceramic systems with 
microcomputed tomography: An in vitro study. J Prosthet Dent 2017;118:200-7.

35. Curran P, Cattani-Lorente M, Anselm Wiskott HW, Durual S, Scherrer SS. Grinding damage assessment for CAD-CAM restorative materials. Dent Mater 2017;33:294-308.

36. Ludovichetti FS, Trindade FZ, Adabo GL, Pezzato L, Fonseca RG. Effect of grinding and polishing on the roughness and fracture resistance of cemented CAD-CAM monolithic materials submitted to mechanical aging. J Prosthet Dent 2019;121:866.e1-8.

37. Coldea A, Fischer J, Swain MV, Thiel N. Damage tolerance of indirect restorative materials (including PICN) after simulated bur adjustments. Dent Mater 2015;31:684-94.

38. Song TJ, Kwon TK, Yang JH, Han JS, Lee JB, Kim SH, Yeo IS. Marginal fit of anterior 3-unit fixed partial zirconia restorations using different CAD/CAM systems. J Adv Prosthodont 2013;5:219-25. 